\section{Perception of inflation: Polynomial not exponential}

\author{
GREGORY V. JONES \\ University of Bristol, Bristol, England
}

In a recent article, Keren (1983) analyzed patterns of misperception of exponential growth. It is argued here, however, that Keren's theoretical analysis is, in fact, inappropriate, and it is shown that an alternative model accounts better for Keren's results. In the first section that follows, Keren's analysis and its problems are reviewed; in the following sections, the alternative model and its empirical application are briefly described.

\section{Misparametricized Exponential Model}

Keren's study derives both theoretically and methodologically from a series conducted by Wagenaar and his colleagues (e.g., Wagenaar \& Sagaria, 1975; Wagenaar \& Timmers, 1978). In these, a subject was typically presented with a series of numbers that were exponentially distributed, and asked to extrapolate from them. It was reliably found that subjective extrapolations were considerably below normative exponential values. For example, confronted with the series $3,7,20,55$, and 148 , subjective extrapolations for its magnitude after a further five units were considerably below the value of 22,026 yielded by the function $y=\exp (x)$. Wagenaar and his colleagues concluded that subjective extrapolations deviated considerably from "correct" ones, and proposed that this occurred because subjects misperceived a function's parameter values. According to their misparametricized exponential theory, $\exp (x)$ is misperceived as $\alpha \exp (\beta x)$. It was pointed out by Jones (1979), however, that no single form of extrapolation can be said to be "correct" in the absence of explicit instructions concerning the nature of the function according to which the initial values of the series are generated. Since explicit generating functions were not, in fact, included by Wagenaar and his colleagues, exponential extrapolations had no special status compared with any other form of extrapolation, including that chosen by subjects. Thus, Keren (1983) is mistaken in commencing his article by stating that "Wagenaar and his coworkers ... have shown that exponential growth ... is misperceived and grossly underestimated by human subjects"' (p. 289).

In the experiments he himself conducted, however, Keren was sensitive to the issue of providing subjects' instructions with explicit generating functions (see footnote 3 of Keren, 1983). As an example, in his Experiment 1 , the subjects were told in one condition not only

Requests for reprints should be sent to Gregory V. Jones, Department of Psychology, University of Bristol, 8-10 Berkeley Square, Bristol BS8 $1 \mathrm{HH}$, England. that the cost of an object in the last 4 years was $\$ 0.61$, $\$ 1.04, \$ 1.76$, and $\$ 3.00$, but also explicitly that the rate of inflation over this period was $70 \%$. Their task was to predict the price in a further 13 years' time, assuming the same rate of inflation. The correct answer here is, in general

$$
Y_{n}=Y_{0}[1+(p / 100)]^{n},
$$

where $Y_{n}$ is the price after $n$ years, $Y_{0}$ the price in the current year, and $p$ the rate of inflation. For $Y_{0}$ and $p$ equal to 3 and 70 , respectively, the predicted exponential value in 13 years' time is $\$ 2,971.4$. However, the median value estimated by subjects was well under a hundredth of this, at $\$ 20.7$.

To account for this huge mismatch, Keren adopted the model of Wagenaar and his colleagues, and proposed that "subjects underestimate the exponent of the function by a factor $\beta$, and attempt to 'correct' for it (insufficiently, of course) by multiplying by a factor $\alpha$ " (Keren, 1983, p. 291), yielding a subjective extrapolation of

$$
\hat{\mathrm{Y}}_{\mathrm{n}}=\alpha \mathrm{Y}_{0}[1+(\mathrm{p} / 100)]^{\beta \mathrm{n}} \text {. }
$$

In effect, it is theorized that subjects utilize the correct equation but plug in the wrong values for the initial price, $\mathrm{Y}_{0}$, and the number of years, $\mathrm{n}$. In the example under discussion, the values of price and of years have to be changed from 3 and 13, respectively, to 5.73 and 2.43 , respectively (see Keren, 1983, Table 2).

It can be seen, therefore, that the misparametricized exponential model rests upon the assumption that subjects manipulate correctly the algebraic apparatus of exponential prediction, with their performance marred only by the adoption of incorrect parameter values. Unfortunately, it is apparent that this assumption is almost certainly incorrect. The subject is explicitly provided with the correct values of all the exponential parameters, and has no reason to adopt other, incorrect ones. The apparatus of exponential prediction, on the other hand, involves formal, abstract paths of analysis, along which there is no evidence to suggest that the subject proceeds. The conclusion, therefore, is that the subject does not insert incorrect parameter values into the intended exponential apparatus of prediction, but instead forms predictions on the basis of a qualitatively different perception of the initial series. This view is espoused by the generalized polynomial model.

\section{Generalized Polynomial Model}

According to this model (Jones, 1979), subjects perceive and extrapolate from series relative to the simplest appropriate polynomial function. In the case of a monotonically accelerating series, as used by Keren (1983), the relevant polynomial is the quadratic. It was shown by 
Jones (1979) that subjective extrapolations over a range of series of differing magnitudes conformed well to this benchmark, consistently falling relatively closely below the best-fitting least squares quadratic line.

Consider, again, the example of the series $\$ 0.61, \$ 1.04$, $\$ 1.76, \$ 3.00$, for which exponential extrapolation yields a value after a further 13 years of $\$ 2,971.4$, very much greater than the median observed value of $\$ 20.7$. Quadratic extrapolation yields a value, $\$ 55.4$, much closer to the median observed one, consistent with the hypothesis that subjects' behavior is based on the imperfectly estimated best-fitting second-order polynomial. ${ }^{1}$

\section{Cultural Differences in the \\ Perception of Growth}

The subjects in the experiment referred to in the preceding sections were from Canada, and had experienced only low inflation in previous years. In two subsequent experiments, however, Keren (1983) tested subjects who were from Israel, and had experienced high inflation in recent years. If everyday experience is important in affecting people's perception of financial inflation, the generalized polynomial model makes a strong qualitative prediction. The subjects in the later experiments should have learned that values may rise with inflation to a much greater extent than expected on the basis of the polynomial benchmark. Accordingly, it is predicted that a crossover in the pattern of results should occur, in that this learning experience may be expected to lead to extrapolations in the later experiments which lie consistently above the polynomial level instead of below it.

Figures 1 and 2 contrast the results in the first experiment and in the later experiments, for series with inflation rates of $13 \%$ and $70 \%$, respectively. The line drawn through the ordinates of Figures 1 and 2 at the value zero

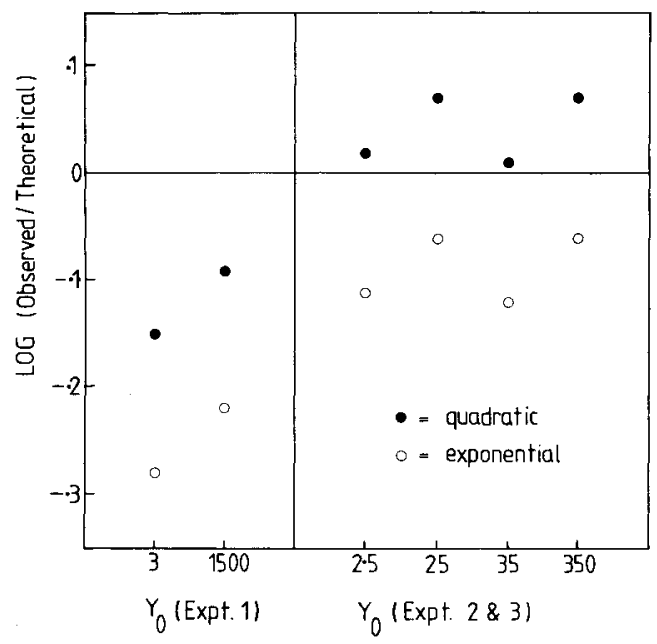

Figure 1. The relation between observed extrapolations and those expected on either a best-fitting quadratic or exponential basis, for current values as shown and inflation at $13 \%$.

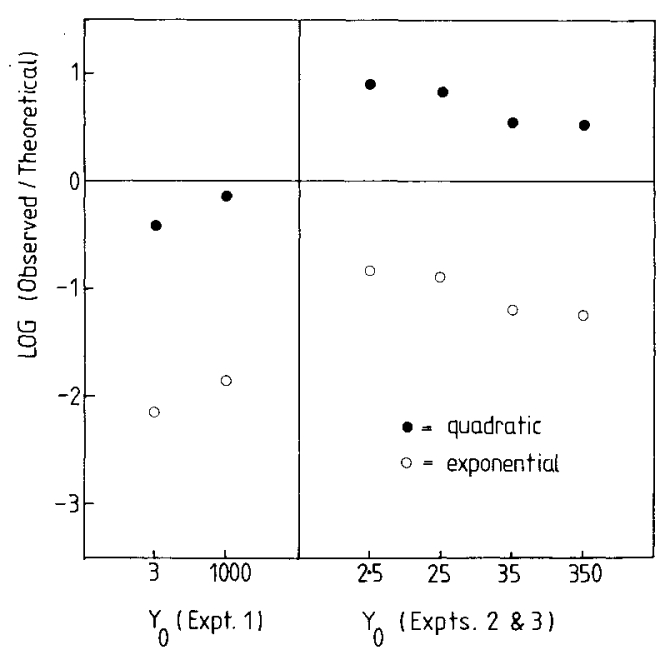

Figure 2. The relation between observed extrapolations and those expected on either a best-fitting quadratic or exponential basis, for current values as shown and inflation at $\mathbf{7 0 \%}$.

represents a perfect match between observed and normative prediction. In both figures, it can be seen that the crossover prediction is confirmed. Observed extrapolations uniformly change from being below quadratic values in the first experiment to being above them in the later experiments.

In contrast, the misparametricized exponential model makes no comparable strong prediction. As can be seen in Figures 1 and 2, observed extrapolations are merely overpredicted somewhat less by exponential values in the later experiments than in the first experiment. Alternatively described, in the later experiments the values of the exponential parameters hypothesized to be substituted for those explicitly presented are decreased in the case of initial price and increased in the case of number of years (see Keren, 1983, Table 2). The interpretation to be accorded this movement of the two parameters in opposite directions is not apparent.

\section{Conclusion}

It is concluded that perception of, and prediction from, series of values conforming to a constant rate of inflation does not involve correct apprehension of the abstract form of exponential growth linked with incorrect perception of parameter values, as assumed by Keren (1983). Instead, it was shown that empirical data are consistent with the hypothesis (Jones, 1979) that the subject's perception of such growth is based upon the simple quadratic.

\section{REFERENCES}

JONES, G. V. (1979). A generalized polynomial model for perception of exponential series. Perception \& Psychophysics, 25, 232-234.

KEREN, G. (1983). Cultural differences in the misperception of exponential growth. Perception \& Psychophysics, 34, 289-293. 
WagenaAR, W. A., \& SAGaria, S. (1975). Misperception of exponential growth. Perception \& Psychophysics, 18, 416-422.

WagenaAr, W. A., \& Timmers, H. (1978). Extrapolation of exponential time series is not enhanced by having more data points. Perception \& Psychophysics, 24, 182-184.

\section{NOTE}

1. The least squares quadratic here is $0.2025 \mathrm{x}^{2}-0.2235 \mathrm{x}+0.6425$. It yields values for $x=1$ to 4 of $\$ 0.62, \$ 1.01, \$ 1.79$, and $\$ 2.99$, respectively.

(Manuscript received June 15, 1984;

accepted for publication June 25, 1984.)

\section{ANNOUNCEMENT \\ Third European Conference on Eye Movements (ECEM3) September 24-27, 1985, Dourdan (near Paris), France}

The Third European Conference on Eye Movements will be held at Dourdan, near Paris, France, September 24-27, 1985. Its aim is to confront work being done in different fields (psychology, physiology, neuropsychology, ophthalmology, engineering, artificial intelligence, ergonomics, pathology, development, education) concerning the functions, capacities, and limitations of the visual system at work through its mobility. Specialized symposia will be held on topics concerning, among others, oculomotor control, eye movements in perception and reading, and eye movements as a tool in ergonomics.

Abstracts for the conference are due in March 1985. For further information, please contact the organizers: Ariane Lévy-Schoen and Kevin O'Regan, Groupe Regard, Laboratoire de Psychologie Expérimentale, 28 rue Serpente, 75006 Paris, France. 